\title{
Genetic Effects of Welding Fumes on the Progression of Neurodegenerative Diseases
}

\author{
Humayan Kabir Rana ${ }^{\mathrm{a}}$, Mst. Rashida Akhtar ${ }^{\mathrm{b}}$, Md Bashir Ahmed $^{\mathrm{c}}$, Pietro Lio'd, Julian \\ M.W. Quinn ${ }^{\mathrm{e}}$, Fazlul Huq ${ }^{\mathrm{f}}$, Mohammad Ali Moni ${ }^{\mathrm{e}, \mathrm{f}, *}$ \\ ${ }^{a}$ Department of Computer Science and Engineering, Green University of Bangladesh \\ ${ }^{b}$ Department of Computer Science and Engineering, Varendra University, Rajshahi, Bangladesh \\ ${ }^{c}$ School of Civil and Environmental Engineering, University of Technology Sydney, NSW 2007, Australia \\ ${ }^{d}$ Computer Laboratory, The University of Cambridge, 15 JJ Thomson Avenue, Cambridge, UK \\ ${ }^{e}$ Bone Biology Division, Garvan Institute of Medical Research, Darlinghurst, NSW, Australia \\ ${ }^{f}$ School of Biomedical Science, Faculty of Medicine and Health, The University of Sydney, Australia
}

\begin{abstract}
Background: Welding exposes different types of fumes, gases and radiant energy that can be potentially dangerous for unsafe welder's health. Welding fumes (WFs) are a significant problem among all those exposed. WFs are a complex mixture of metallic oxides, silicates and fluorides that may result in different health effects. If a welder inhales such fumes in large quantities over a long period of time, there is a risk of various neurodegenerative diseases (NDGDs) development.

Methods: We developed quantitative frameworks to identify the genetic relationship of WFs and NDGDs. We analyzed Gene Expression microarray data from WFs exposed tissues and NDGDs including Parkinson's disease (PD), Alzheimer's disease (AD), Lou Gehrig's disease (LGD), Epilepsy disease (ED), Multiple Sclerosis disease (MSD) datasets. We constructed disease-gene relationship networks and identified dysregulated pathways, ontological pathways and protein-protein interaction sub-network using multilayer network topology and neighborhood-based benchmarking.

Results: We observed that WFs shares 18, 16, 13, 19 and 19 differentially expressed genes with PD, AD, LGD, ED and MSD respectively. Gene expression dysregulation along with relationship networks, pathways and ontologic analysis showed that WFs are responsible for the progression of $\mathrm{PD}, \mathrm{AD}, \mathrm{LGD}, \mathrm{ED}$ and MSD neurodegenerative diseases.

Conclusion: Our developed network-based approach to analysis and investigate the genetic effects of welding fumes on PD, AD, LGD, ED and MSD neurodegenerative diseases could be helpful to understand the causal influences of WF exposure for the progression of the NDGDs.
\end{abstract}

Keywords: Welding fumes, Alzheimer's disease, Parkinson's disease, Epilepsy disease, Neurodegenerative diseases.

\footnotetext{
*Corresponding author:

Email address: mohammad.moni@sydney.edu.au (Mohammad Ali Moni)
} 


\section{Introduction}

Welding process is very dangerous because it exposes different types of fumes, gases and radiant energy. Welding fumes (WFs) are the most venturous partial among all welding exposers [1]. WFs are an intricate mixture of metallic oxides, silicates and fluorides include Beryllium, Aluminum, Cadmium, Chromium, Copper, Iron, Lead, Manganese, Magnesium, Nickel, Vanadium, and Zinc etc. [2]. If a welder inhales welding fumes in large quantities over a long period of time, this may convey various NDGDs $[1,3]$.

Neurodegenerative diseases (NDGDs) are a collective term for a heterogeneous group of disorders that are incurable and characterized by the progressive degeneration of the function and structure of the central nervous system [4]. NDGDs primarily attack the neurons of the central nervous system and progressively damage the function of them. Neurons are most vulnerable to injury and normally dont reproduce or replace themselves [5]. If neurons become damaged or die they cannot be replaced by medical treatments. So that NDGDs are very dangerous and currently they dont have any cure. We studied several NDGDs include $\mathrm{PD}, \mathrm{AD}, \mathrm{LGD}, \mathrm{ED}$ and MSD to find the effects of welding fumes on them.

$\mathrm{PD}$ is the second-most neurologic disease that affects neural cells in the brain which produce dopamine in the substantia nigra [6]. There are several symptoms of PD include tremors, muscle rigidity, and changes in gait and speech. Welding fumes contain Manganese that can develop Parkinsons disease [7]. The AD is the most common type of incurable dementia that causes problems with progressive memory loss and other cognitive abilities. Existing medical treatments for AD produce only a modest improvement of symptoms but there is currently no cure [8]. Aluminum exposure to welding is a risk factor to produce AD. LGD also cognizant as Amyotrophic lateral sclerosis (ALS), is a neurodegenerative disease that progressively damages motor neurons and muscle atrophy controlling voluntary muscle movement. The initial symptoms of LGD are muscle weakness or stiffness, can bring death by progressive muscular paralysis and respiratory system failure within 2 to 5 years. US Food and Drug Administration (FDA) approved Riluzole and Edaravone drugs that could prolong LGD survival. But still, there is no effective cure or prevention for this devastating disease $[9,10]$. ED is a heterogeneous group of neurodegenerative disorder that affects neural cells in the brain which are recognized by recurrent seizures or unusual behavior, awareness and sensations suffering over 60 million people in the world. AEDs are Current anti-epileptic drugs that can minimize symptoms but there is no permanent cure or prevention of ED [11]. MSD is a devastating neurodegenerative disorder that attacks the neurons of the central nervous system in the spinal cord and brain, on young adults most commonly [12]. The symptom of MSD can be included as muscle weakness, trouble with sensation, blindness in one eye or double vision. Medical treatments of MSD could prolong only survival but there is no permanent cure or prevention of MSD. Manganese exposure to welding is a main risk factor on the progression of LGD, ED and MSD [13].

Our study employed a systematic and quantitative approach to find the genetic effects of WFs on NDGDs. For these purposes, we studied several NDGDs including PD, AD, LGD, ED and MSD. To understand the effects of WFs on NDGDs, we examined gene expression dysregulation, disease association network, dysregulated pathway, gene expression ontology 
and protein-protein interaction. We also investigated the validation of our study by using the gold benchmark databases (dbGAP and OMIM).

\section{Materials and Methods}

\subsection{Datasets employed in this study}

To investigate the effects of WFs on NDGDs at the molecular level, we used gene expression microarray data. In this study, we used Gene Expression Omnibus from the National Center for Biotechnology Information (NCBI) (http://www.ncbi.nlm.nih.gov/geo/). We analyzed 6 different datasets for our study with accession numbers GSE62384, GSE19587, GSE28146, GSE833, GSE22779 and GSE38010 [14, 15, 16, 17, 18, 19]. The WFs dataset (GSE62384) is a result of gene expression analysis of fresh welding fumes influence on upper airway epithelial cells (RPMI 2650). This Data is collected from the people with sparkgenerated welding fumes at high $(760 \mathrm{~g} / \mathrm{m} 3)$ and low $(85 \mathrm{~g} / \mathrm{m} 3)$ concentrations. The donors inhaled welding fumes for 6 hours continuously, followed by zero hours or four hours postexposure incubation. The PD dataset (GSE19587) is taken from 6 postmortem brains of PD patients and from 5 control brains. The AD dataset (GSE28146) is a microarray data on RNA from fresh frozen hippocampal tissue blocks that contain both white and gray matter, potentially obscuring region-specific changes. The LGD dataset (GSE833) is an Affymetrix Human Full Length HuGeneFL [Hu6800] Array. In this data, postmortem spinal cord grey matter from sporadic and familial ALGD patients are compared with controls. The ED dataset (GSE22779) is a gene expression profiles of 4 non-leukemic individuals (1 healthy and 3 with epilepsy) is generated from the mononuclear cells isolated from the peripheral blood samples before, and after 2, 6, and 24 hours of in-vivo glucocorticoid treatment. The MSD dataset (GSE38010) is a microarray data of multiple sclerosis (MS) patients brain lesions compared with control brain samples.

\subsection{Overview of analytical approach}

We used systematic and quantitative approach to identify the effect of WFs on the progression of the NDGDs using different sources of available microarray datasets. The graphical representation of this approach is shown in figure 1. This approach included gene expression, signaling pathway, Gene Ontology (GO) and protein-protein interaction analyses. This approach also used Gold benchmark data to verify the validity of our study.

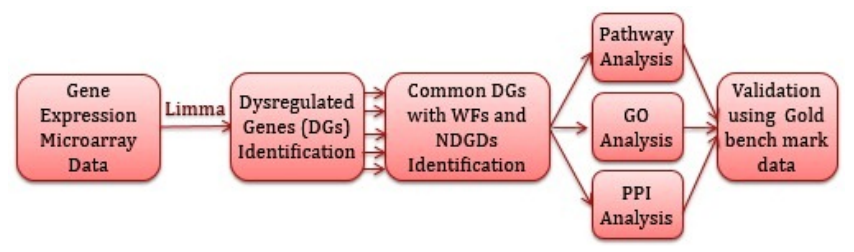

Figure 1: Flow-diagram of the analytical approach used in this study. 


\subsection{Analysis methods}

Gene expression analysis using microarrays is a global and popular method to develop and refine the molecular determinants of human disorders that have proven to be a sensitive method [20]. We used these technologies to analyze the gene expression profiles of Parkinson's disease (PD), Alzheimer's disease (AD), Lou Gehrig's disease (LGD), Epilepsy disease (ED) and Multiple Sclerosis disease (MSD) to find the effects of welding fumes on them. To uniform the mRNA expression data of different platforms and to avoid the problems of experimental systems, we normalized the gene expression data (disease state or control data) by using the Z-score transformation $\left(Z_{i j}\right)$ for each NDGD gene expression profile using $Z_{i j}=\frac{g_{i j}-\operatorname{mean}\left(g_{i}\right)}{S D\left(g_{i}\right)}$,

where $S D$ implies the standard deviation, $g_{i j}$ represents the value of the gene expression $i$ in sample $j$. After this transformation we can directly compare of gene expression values of various diseases under different platforms. We applied two conditions for t-test statistic. We performed unpaired T-test to identify differentially expressed genes in patients over control data and selected significant genes. We have chosen a threshold of at least $1 \log _{2}$ fold change and a $p$-value of $<=1 * 10^{-2}$.

We applied the topological methods and neighborhood based benchmark to find genedisease associations. Gene-disease network (GDN) was constructed by using the gene-disease associations, where the nods in the network represent either gene or disease. This network can also be characterized as a bipartite graph. The diseases are connected in GDN when they share at least one or more significant differentially expressed genes. Let $D$ is a specific set of diseases and $G$ is a set of dysregulated genes, gene-disease associations attempt to find whether gene $g \in G$ is associated with disease $d \in D$. If $G_{i}$ and $G_{j}$, the sets of significant dysregulated genes associated with diseases $D_{i}$ and $D_{j}$ respectively, then the number of shared dysregulated genes $\left(n_{i j}^{g}\right)$ associated with both diseases $D_{i}$ and $D_{j}$ is as follows [20]:

$$
n_{i j}^{g}=N\left(G_{i} \cap G_{j}\right)
$$

The common neighbours are the based on the Jaccard Coefficient method, where the edge prediction score for the node pair is as [20]:

$$
E(i, j)=\frac{N\left(G_{i} \cap G_{j}\right)}{N\left(G_{i} \cup G_{j}\right)}
$$

where $G$ is the set of nodes and $E$ is the set of all edges. We used R software packages "comoR" [21] and "POGO" [22] to cross check the genes-diseases associations.

To find molecular pathways of several NDGDs, we have analyzed pathway and gene ontology using Enrichr (https : //amp.pharm.mssm.edu/Enrichr/), a comprehensive gene set enrichment analysis web-based tool. We used STRING (https ://string $-d b . o r g$.$) for$ analyzing protein-protein interactions.

\section{Results}

\subsection{Gene Expression Analysis}

To investigate the effects of WFs on NDGDs, we analyzed the gene expressing microarray data from the National Center for Biotechnology Information (NCBI) (http : 
//www.ncbi.nlm.nih.gov/geo/). We found that 903 genes were differentially expressed for WFs with adjusted $P<=.01$ and $|\log F C|>=1$. Among them, 392 and 511 were up and down regulated respectively. Similarly, our analysis identified the most significant differentially expressed genes for each NDGD after various steps of statistical analysis. We identified differentially expressed genes, 774 (263 up and 511 down) in PD, 565 (291 up and 274 down) in AD, 501 (296 up and 205 down) in LGD, 725 (350 up and down) in ED and 834 (455 up and 388 down) in MSD. The cross-comparative analysis was also performed to find the common differentially expressed genes between WFs and each NDGD. We observed that WFs shares 18, 16, 13, 19 and 19 differentially expressed genes with PD, AD, LGD, ED and MSD respectively. To find the significant associations among these NDGDs with WFs, we built two separate disease relationships networks for up and down-regulated genes, centered on the WFs as shown in figure 2 and 3. Two diseases are associated with each if there exist one or more common genes in between these diseases [23]. Noticeably, 2 significant genes, N4BD2L2 and NAAA are commonly differentially expressed among WFs, LGD and WD; one gene DAAM1 is commonly dysregulated among WFs, ED and MSD.

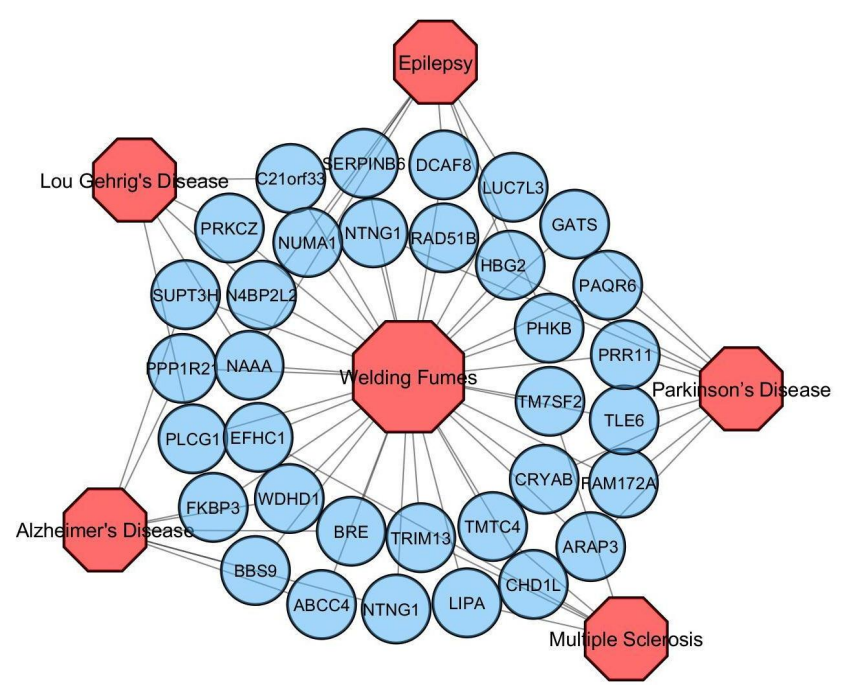

Figure 2: Disease network of Welding fumes (WFs) with Parkinson's disease (PD), Alzheimer's disease (AD), Lou Gehrig's disease (LGD), Epilepsy disease (ED) and Multiple Sclerosis disease (MSD). Red colored octagon-shaped nodes represent different categories of disease, and round-shaped sky blue colored nodes represent commonly up-regulated genes for WFs with the other neurodegenerative disorders. A link is placed between a disorder and a disease gene if mutations in that gene lead to the specific disorder.

\subsection{Pathway and Functional Association Analysis}

Pathways are the key to know how an organism reacts to perturbations in its internal changes. The pathway-based analysis is a modern technique to understand how different complex diseases are related to each other by underlying molecular or biological mechanisms [24]. We analyzed pathways of the common differentially expressed genes using Enrichr, a comprehensive gene set enrichment analysis web-based tool [25]. Pathways of the commonly dysregulated genes in between WFs and each NDGD were analyzed using four databases 


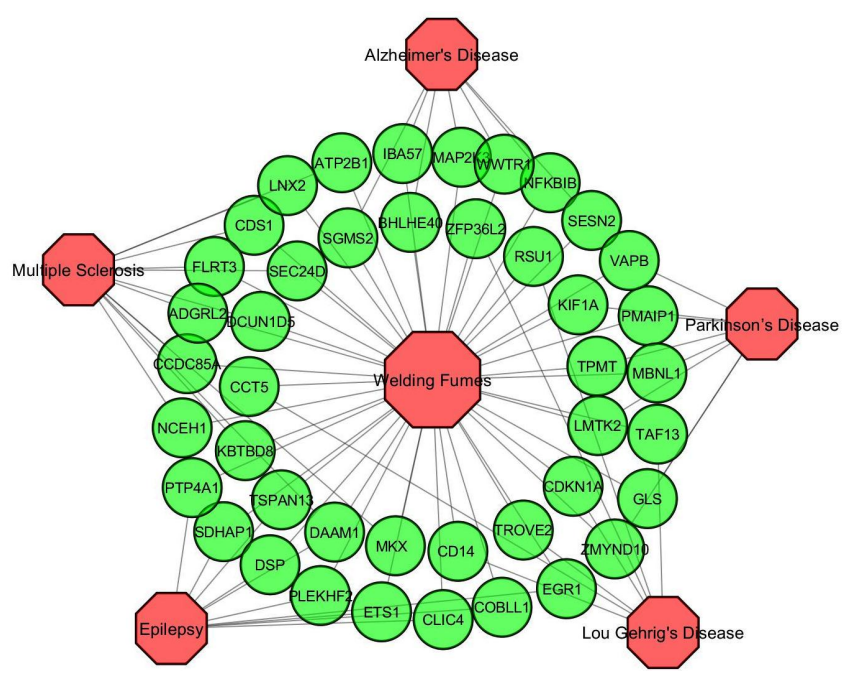

Figure 3: Disease network of Welding fumes (WFs) with Parkinson's disease (PD), Alzheimer's disease (AD), Lou Gehrig's disease (LGD), Epilepsy disease (ED) and Multiple Sclerosis disease (MSD). Red colored octagon-shaped nodes represent different categories of disease, and round-shaped green colored nodes represent commonly down-regulated genes for WFs with the other neurodegenerative disorders. A link is placed between a disorder and a disease gene if mutations in that gene lead to the specific disorder.

includes KEGG, WikiPathways, Reactome and BioCarta. We combined pathways from four mentioned databases and identified the most significant pathways of each disease after various steps of statistical analysis.

We observed that PD has five significant pathways as shown in table 1. Among these pathways, 'Glutamate Neurotransmitter Release Cycle' is responsible to release the glutamate from the presynaptic neuron and its binding to glutamate receptors on the postsynaptic cell to generate a series of events that lead to the propagation of the synaptic transmission [26]. The pathway 'Sphingolipid de novo biosynthesis' is responsible to provide signals in molecules that regulate various biological functions [27]. The pathway 'Intrinsic Pathway for Apoptosis' is responsible to manage a variety of intracellular stress signal including DNA damage, growth factor withdrawal, unfolding stresses in the endoplasmic reticulum and death receptor stimulation [28]. Kinesins are a super-group of motor proteins based on microtubule that has various functions in the transport of vesicles, organelles, chromosomes, and regulate microtubule dynamics [29]. The pathway 'Neurotransmitter Release Cycle' is responsible to control electrical signals passing through the axons in the form of action potential.

We observed that AD has four significant pathways as shown in table 2. Among these pathways, 'Circadian rhythm pathway' is responsible to feed and influence clocks in other tissues by hormone secretion and nervous stimulation from the brain [30]. Sphingomyelin synthesis appears to be regulated primarily at the level of this transport process through the reversible phosphorylation of CERT (Saito et al. 2008). 'Amyotrophic lateral sclerosis (ALS)' is responsible for most common motor neuron disease [31]. 'MAPKinase Signaling Pathway' is responsible for manage signals of reactions that regulate cell proliferation and 
Table 1: Pathways Associated with Significantly Commonly Differentially Expressed Genes of the PD with WFs.

\begin{tabular}{|l|l|l|}
\hline \multicolumn{1}{|c|}{ Pathway } & $\begin{array}{l}\text { Genes in the } \\
\text { pathway }\end{array}$ & $\begin{array}{l}\text { Adjusted } \\
\text { p-value }\end{array}$ \\
\hline Glutamate Neurotransmitter Release Cycle & GLS & $2.02 \mathrm{E}-02$ \\
\hline Sphingolipid De Novo Biosynthesis & VAPB & $2.77 \mathrm{E}-02$ \\
\hline Intrinsic Pathway for Apoptosis & PMAIP1 & $3.51 \mathrm{E}-02$ \\
\hline Kinesins Pathway & KIF1A & $3.68 \mathrm{E}-02$ \\
\hline Neurotransmitter Release Cycle & GLS & $4.25 \mathrm{E}-02$ \\
\hline
\end{tabular}

apoptosis [32].

Table 2: Pathways Associated with Significantly Commonly Differentially Expressed Genes of the AD with WFs.

\begin{tabular}{|l|l|l|}
\hline \multicolumn{1}{|c|}{ Pathway } & $\begin{array}{l}\text { Genes in the } \\
\text { pathway }\end{array}$ & $\begin{array}{l}\text { Adjusted } \\
\text { p-value }\end{array}$ \\
\hline Circadian rhythm pathway & BHLHE40 & $2.23 \mathrm{E}-02$ \\
\hline Sphingolipid metabolism pathway & SGMS2 & $3.47 \mathrm{E}-02$ \\
\hline Amyotrophic lateral sclerosis (ALS) & MAP2K3 & $3.76 \mathrm{E}-02$ \\
\hline MAPKinase Signaling Pathway & MAP2K3 & $4.12 \mathrm{E}-02$ \\
\hline
\end{tabular}

We observed that LGD has six significant pathways as shown in table 3. Among these pathways, 'Rap1 signaling pathway' is responsible for controlling a variety of processes, such as cell adhesion, cell polarity and cell-cell junction formation [33]. 'P53 signaling pathway' manages various stress signals, including activated oncogenes, oxidative stress and DNA damage.

Table 3: Pathways Associated with Significantly Common Differentially Expressed Genes of the LGD with WFs.

\begin{tabular}{|l|l|l|}
\hline \multicolumn{1}{|c|}{ Pathway } & $\begin{array}{l}\text { Genes in the } \\
\text { pathway }\end{array}$ & $\begin{array}{l}\text { Adjusted } \\
\text { p-value }\end{array}$ \\
\hline Signaling Pathways in Glioblastoma & $\begin{array}{l}\text { CDKN1A, } \\
\text { PLCG1, PRKCZ }\end{array}$ & $1.48 \mathrm{E}-05$ \\
\hline MAPK Signaling Pathway & CD14;PRKCZ & $4.38 \mathrm{E}-03$ \\
\hline TRIF-mediated programmed cell death & CD14 & $5.99 \mathrm{E}-03$ \\
\hline EPO Signaling Pathway & PLCG1 & $6.58 \mathrm{E}-03$ \\
\hline Rap1 signaling pathway & PLCG1, PRKCZ & $6.82 \mathrm{E}-03$ \\
\hline P53 signaling pathway & CDKN1A & $4.06 \mathrm{E}-02$ \\
\hline
\end{tabular}

We observed that ED has five significant pathways as shown in table 4. Among these pathways, 'Neurotransmitter Release Cycle' is responsible to control electrical signals passing through the axons in the form of action potential. 'Glycogen Metabolismserves' serves as a major stored fuel for several tissues. The keratinocytes function is to form a barrier against environmental damage by fungi pathogenic bacteria, parasites, viruses, and UV radiation.

We observed that MSD has five significant pathways as shown in table 5. Among these pathways, 'Endocrine and other factor-regulated calcium reabsorption' is essential for numerous physiological functions including muscle contraction, intracellular signalling processes, neuronal excitability and bone formation [34]. 'Mineral absorption' provides mineral in the 
Table 4: Pathways Associated with Significantly Common Differentially Expressed Genes of the ED with WFs.

\begin{tabular}{|l|l|l|}
\hline \multicolumn{1}{|c|}{ Pathway } & $\begin{array}{l}\text { Genes in the } \\
\text { pathway }\end{array}$ & $\begin{array}{l}\text { Adjusted p- } \\
\text { value }\end{array}$ \\
\hline Ectoderm Differentiation & $\begin{array}{l}\text { NUMA1, } \\
\text { SERPINB6 }\end{array}$ & $6.82 \mathrm{E}-03$ \\
\hline NR3C Signaling & EGR1 & $1.70 \mathrm{E}-02$ \\
\hline Glycogen Metabolism & PHKB & $3.19 \mathrm{E}-02$ \\
\hline Neurotransmitter Release Cycle & NAAA & $4.49 \mathrm{E}-02$ \\
\hline Keratinocyte Differentiation & ETS1 & $4.67 \mathrm{E}-02$ \\
\hline
\end{tabular}

neural cell to sustain life. 'Cholesterol biosynthesis' controls cholesterol to the nucleus and activating genes.

Table 5: Pathways Associated with Significantly Common Differentially Expressed Genes of the MSD with WFs.

\begin{tabular}{|l|l|l|}
\hline \multicolumn{1}{|c|}{ Pathway } & $\begin{array}{l}\text { Genes in the } \\
\text { pathway }\end{array}$ & $\begin{array}{l}\text { Adjusted p- } \\
\text { value }\end{array}$ \\
\hline Steroid biosynthesis & LIPA;TM7SF2 & $1.44 \mathrm{E}-04$ \\
\hline Metabolism of lipids and lipoproteins & $\begin{array}{l}\text { CDS1, NCEH1, } \\
\text { SEC24D, TM7SF2 }\end{array}$ & $2.47 \mathrm{E}-03$ \\
\hline Cholesterol biosynthesis & TM7SF2 & $2.05 \mathrm{E}-02$ \\
\hline $\begin{array}{l}\text { Endocrine and other factor-regulated calcium } \\
\text { reabsorption }\end{array}$ & ATP2B1 & $4.15 \mathrm{E}-02$ \\
\hline Mineral absorption & ATP2B1 & $4.49 \mathrm{E}-02$ \\
\hline
\end{tabular}

\subsection{Gene Ontological Analysis}

The Gene Ontology (GO) refers to a universal conceptual model for representing gene functions and their relationship in the domain of gene regulation. It is constantly expanded by accumulating the biological knowledge to cover regulation of gene functions and the relationship of these functions in terms of ontology classes and semantic relations between classes [35]. GO of the significantly dysregulated genes were analyzed using Enrichr, a comprehensive gene set enrichment analysis web-based tool [25]. GO of the commonly differentially expressed genes (i.e. Dysregulated genes in between WFs and each NDGD) for each NDGD and WFs were analyzed using two databases of Enrichr including GO Biological Process and Human Phenotype Ontology. We combined ontologies from two mentioned databases and identified the most significant GO term of each disease after various steps of statistical analysis. We observed that 15, 15, 24, 19 and 17 gene ontology classes are associated with the significantly commonly dysregulated (i.e. Dysregulated genes in between WFs and each NDGD) genes for WFs with the PD, AD, LGD, Ed and MSD respectively as shown in table 6-10.

\subsection{Protein-Protein Interaction Analysis}

Protein-protein interaction networks (PPINs) are the mathematical representation of the physical contacts of proteins in the cell. Protein-protein interactions (PPIs) are essential to every molecular and biological process in a cell, so PPIs is crucial to understand cell 
bioRxiv preprint doi: https://doi.org/10.1101/480806; this version posted December 2, 2018. The copyright holder for this preprint (which was not certified by peer review) is the author/funder, who has granted bioRxiv a license to display the preprint in perpetuity. It is made available under aCC-BY-NC-ND 4.0 International license.

Table 6: Gene Ontologies Associated with the Significantly Commonly Dysregulated Genes of the PD with WFs.

\begin{tabular}{|l|l|l|l|}
\hline G0 Term & \multicolumn{1}{|c|}{ Pathway } & $\begin{array}{l}\text { Genes in the } \\
\text { Pathway }\end{array}$ & $\begin{array}{l}\text { ldjusted p- } \\
\text { value }\end{array}$ \\
\hline G0:0001844 & $\begin{array}{l}\text { Protein insertion into mitochondrial membrane } \\
\text { involved in apoptotic signaling pathway }\end{array}$ & PMAIP1 & $5.94 \mathrm{E}-03$ \\
\hline GO:1902043 & $\begin{array}{l}\text { Positive regulation of extrinsic apoptotic signaling } \\
\text { pathway via death domain receptors }\end{array}$ & PMAIP1 & $5.94 \mathrm{E}-03$ \\
\hline GO:0006987 & $\begin{array}{l}\text { Activation of signaling protein activity involved in } \\
\text { unfolded protein response }\end{array}$ & VAPB & $6.78 \mathrm{E}-03$ \\
\hline G0:0001881 & Receptor recycling & LMTK2 & $8.47 \mathrm{E}-03$ \\
\hline GO:0045837 & Negative regulation of membrane potential & PMAIP1 & $5.94 \mathrm{E}-03$ \\
\hline GO:0043029 & T cell homeostasis & PMAIP1 & $9.31 \mathrm{E}-03$ \\
\hline G0:0032463 & Negative regulation of protein homooligomerization & CRYAB & $7.63 \mathrm{E}-03$ \\
\hline G0:0032075 & Positive regulation of nuclease activity & VAPB & $9.31 \mathrm{E}-03$ \\
\hline G0:0016192 & Vesicle-mediated transport & LMTK2, ARAP3, & $4.73 \mathrm{E}-03$ \\
\hline GO:1905906 & Regulation of amyloid fibril formation & CRYAB & $7.63 \mathrm{E}-03$ \\
\hline HP:0003677 & Slow progression & KIF1A, CRYAB & $3.08 \mathrm{E}-03$ \\
\hline HP:0007210 & Lower limb amyotrophy & KIF1A & $8.47 \mathrm{E}-03$ \\
\hline HP:0200073 & $\begin{array}{l}\text { Respiratory insufficiency due to defective ciliary } \\
\text { clearance }\end{array}$ & ZMYND10 & $7.63 \mathrm{E}-03$ \\
\hline HP:0003323 & Progressive muscle weakness & VAPB & $9.31 \mathrm{E}-03$ \\
\hline HP:0003555 & Muscle fiber splitting & CRYAB & $6.78 \mathrm{E}-03$ \\
\hline
\end{tabular}

Table 7: Gene Ontologies Associated with the Significantly Commonly Dysregulated Genes of the AD with WFs.

\begin{tabular}{|l|l|l|l|}
\hline G0 Term & \multicolumn{1}{|c|}{ Pathway } & $\begin{array}{l}\text { Genes in the } \\
\text { Pathway }\end{array}$ & $\begin{array}{l}\text { Adjusted p- } \\
\text { value }\end{array}$ \\
\hline G0:0043619 & $\begin{array}{l}\text { Regulation of transcription from RNA polymerase II } \\
\text { promoter in response to oxidative stress }\end{array}$ & SESN2 & $6.73 \mathrm{E}-03$ \\
\hline G0:0032055 & $\begin{array}{l}\text { Negative regulation of translation in response to } \\
\text { stress }\end{array}$ & SESN2 & $5.24 \mathrm{E}-03$ \\
\hline G0:0006684 & Sphingomyelin metabolic process & SGMS2 & $7.48 \mathrm{E}-03$ \\
\hline G0:0035414 & Negative regulation of catenin import into nucleus & WWTR1 & $7.48 \mathrm{E}-03$ \\
\hline G0:1990253 & Cellular response to leucine starvation & SESN2 & $8.22 \mathrm{E}-03$ \\
\hline G0:0032309 & Icosanoid secretion & ABCC4 & $8.97 \mathrm{E}-03$ \\
\hline G0:0045859 & Regulation of protein kinase activity & MAP2K3, & $5.36 \mathrm{E}-03$ \\
\hline HP:0001156 & Brachydactyly syndrome & NWTNG1, BBS9 & $8.87 \mathrm{E}-03$ \\
\hline HP:0002141 & Gait imbalance & BBS9 & $1.34 \mathrm{E}-02$ \\
\hline HP:0007707 & Congenital primary aphakia & BBS9 & $1.34 \mathrm{E}-02$ \\
\hline HP:0010747 & Medial flaring of the eyebrow & BBS9 & $1.56 \mathrm{E}-02$ \\
\hline HP:0009806 & Nephrogenic diabetes insipidus & BBS9 & $1.49 \mathrm{E}-02$ \\
\hline HP:0002370 & Poor coordination & BBS9 & $1.71 \mathrm{E}-02$ \\
\hline HP:0006829 & Severe muscular hypotonia & IBA57 & $1.79 \mathrm{E}-02$ \\
\hline HP:0001827 & Genital tract atresia & BBS9 & $1.93 \mathrm{E}-02$ \\
\hline & & & \\
\hline & & &
\end{tabular}

Table 8: Gene Ontologies Associated with the Significantly Commonly Dysregulated Genes of the LGD with WFs.

\begin{tabular}{|l|l|l|l|}
\hline \multicolumn{1}{|c|}{ G0 Term } & \multicolumn{1}{|c|}{ Pathway } & $\begin{array}{l}\text { Genes in the } \\
\text { Pathway }\end{array}$ & $\begin{array}{l}\text { Adjusted p- } \\
\text { value }\end{array}$ \\
\hline G0:2000737 & Negative regulation of stem cell differentiation & $\begin{array}{l}\text { N4BP2L2, } \\
\text { ZFP36L2 }\end{array}$ & $1.81 \mathrm{E}-05$ \\
\hline G0:0034128 & $\begin{array}{l}\text { Negative regulation of MyD88-independent toll-like } \\
\text { receptor signaling pathway }\end{array}$ & CD14 & $4.79 \mathrm{E}-03$ \\
\hline G0:0071364 & Cellular response to epidermal growth factor stimulus & PLCG1, ZFP36L2 & $1.06 \mathrm{E}-04$ \\
\hline G0:1901988 & Negative regulation of cell cycle phase transition & ZFP36L2 & $4.19 \mathrm{E}-03$ \\
\hline G0:1903708 & Positive regulation of hemopoiesis & N4BP2L2 & $4.79 \mathrm{E}-03$ \\
\hline G0:1901991 & $\begin{array}{l}\text { Negative regulation of mitotic cell cycle phase } \\
\text { transition }\end{array}$ & $\begin{array}{l}\text { CDKN1A, } \\
\text { ZFP36L2 }\end{array}$ & $1.02 \mathrm{E}-03$ \\
\hline G0:0071363 & Cellular response to growth factor stimulus & PLCG1, ZFP36L2 & $3.07 \mathrm{E}-03$ \\
\hline G0:0050821 & Protein stabilization & CDKN1A, CCT5 & $3.65 \mathrm{E}-03$ \\
\hline HP:0001738 & Exocrine pancreatic insufficiency & CDKN1A & $1.13 \mathrm{E}-02$ \\
\hline HP:0010832 & Abnormality of pain sensation & CCT5 & $1.49 \mathrm{E}-02$ \\
\hline HP:0002717 & Adrenal overactivity & CDKN1A & $1.79 \mathrm{E}-02$ \\
\hline HP:0003431 & Decreased motor nerve conduction velocity & CCT5 & $1.79 \mathrm{E}-02$ \\
\hline HP:0001258 & Spastic paraplegia & CCT5 & $1.84 \mathrm{E}-02$ \\
\hline HP:0002936 & Distal sensory impairment & CCT5 & $3.66 \mathrm{E}-02$ \\
\hline & & & \\
\hline & & &
\end{tabular}


Table 9: Gene Ontologies Associated with the Significantly Commonly Dysregulated Genes of the ED with WFs.

\begin{tabular}{|l|l|l|l|}
\hline G0 Term & \multicolumn{1}{|c|}{ Pathway } & $\begin{array}{l}\text { Genes in the } \\
\text { Pathway }\end{array}$ & $\begin{array}{l}\text { Adjusted p- } \\
\text { value }\end{array}$ \\
\hline GO:0086069 & Bundle of His cell to Purkinje myocyte communication & DSP & $8.07 \mathrm{E}-03$ \\
\hline GO:0086073 & $\begin{array}{l}\text { Bundle of His cell-Purkinje myocyte adhesion involved } \\
\text { in cell communication }\end{array}$ & DSP & $6.28 \mathrm{E}-03$ \\
\hline GO:0030575 & Nuclear body organization & ETS1 & $7.18 \mathrm{E}-03$ \\
\hline GO:0003223 & Ventricular compact myocardium morphogenesis & DSP & $6.28 \mathrm{E}-03$ \\
\hline GO:0002934 & Desmosome organization & DSP & $8.07 \mathrm{E}-03$ \\
\hline GO:0051639 & Actin filament network formation & COBLL1 & $8.07 \mathrm{E}-03$ \\
\hline GO:1903708 & Positive regulation of hemopoiesis & N4BP2L2 & $7.18 \mathrm{E}-03$ \\
\hline HP:0011902 & Abnormal hemoglobin & HBG2 & $1.25 \mathrm{E}-02$ \\
\hline HP:0001663 & Ventricular fibrillation & DSP & $8.97 \mathrm{E}-03$ \\
\hline HP:0003445 & EMG: neuropathic changes & DCAF8 & $1.79 \mathrm{E}-02$ \\
\hline HP:0011663 & Right ventricular cardiomyopathy & DSP & $8.07 \mathrm{E}-03$ \\
\hline HP:0001730 & Progressive hearing impairment & SERPINB6 & $1.70 \mathrm{E}-02$ \\
\hline & & & \\
\hline
\end{tabular}

Table 10: Gene Ontologies Associated with the Significantly Commonly Dysregulated Genes of the MSD with WFs.

\begin{tabular}{|l|l|l|l|}
\hline G0 Term & \multicolumn{1}{|c|}{ Pathway } & $\begin{array}{l}\text { Genes in the } \\
\text { Pathway }\end{array}$ & $\begin{array}{l}\text { Adjusted p- } \\
\text { value }\end{array}$ \\
\hline G0:0021795 & Cerebral cortex cell migration & EFHC1 & $6.28 \mathrm{E}-03$ \\
\hline G0:0048678 & Response to axon injury & FLRT3 & $1.07 \mathrm{E}-02$ \\
\hline G0:1902187 & Negative regulation of viral release from host cell & TRIM13 & $1.25 \mathrm{E}-02$ \\
\hline G0:0014033 & Neural crest cell differentiation & KBTBD8 & $1.43 \mathrm{E}-02$ \\
\hline G0:0006293 & $\begin{array}{l}\text { Nucleotide-excision repair, preincision complex } \\
\text { stabilization }\end{array}$ & CHD1L & $1.96 \mathrm{E}-02$ \\
\hline HP:0004311 & Abnormality of macrophages & LIPA & $1.70 \mathrm{E}-02$ \\
\hline HP:0001433 & Hepatosplenomegaly & LIPA & $2.23 \mathrm{E}-02$ \\
\hline HP:0100639 & Erectile abnormalities & FLRT3 & $2.84 \mathrm{E}-02$ \\
\hline HP:0002612 & Congenital hepatic fibrosis & LIPA & $3.37 \mathrm{E}-02$ \\
\hline HP:0001522 & Death in infancy & LIPA & $3.98 \mathrm{E}-02$ \\
\hline
\end{tabular}

physiology in disease and healthy states [36]. PPIs of the differentially expressed genes were analyzed using STRING, a biological database and web resource of known and predicted protein-protein interactions [37]. We constructed protein-protein interaction network of significantly commonly dysregulated genes (i.e. Dysregulated genes in between WFs and each NDGD) of all NDGDs using STRING. We clustered into five different groups of interactions of five NDGDs as shown in figure 4.

\section{Discussion}

We investigated the genetic relationship of Welding fumes (WFs) and neurodegenerative diseases (NDGDs) based on the associations of genetics, signaling pathways, gene expression ontologies and protein-protein interactions network. For the purpose of our study, we analyzed Gene Expression Omnibus (GEO) microarray data from WFs, Parkinson's disease (PD), Alzheimer's disease (AD), Lou Gehrig's disease (LGD), Epilepsy disease (ED), Multiple Sclerosis disease (MSD) and control datasets. We found a good number of significantly commonly dysregulated genes in between WFs and NDGDs by gene expression analysis. As there have a good number of significantly commonly dysregulated genes of WFs and NDGDs, it determines that WFs should have effects on NDGDs. Our two separate disease relationships networks for up and down-regulated genes strongly indicated that WFs are highly responsible for NDGDs as shown in Figure 2 and 3. The pathway-based analysis is a modern technique to understand how different complex diseases are related to each 


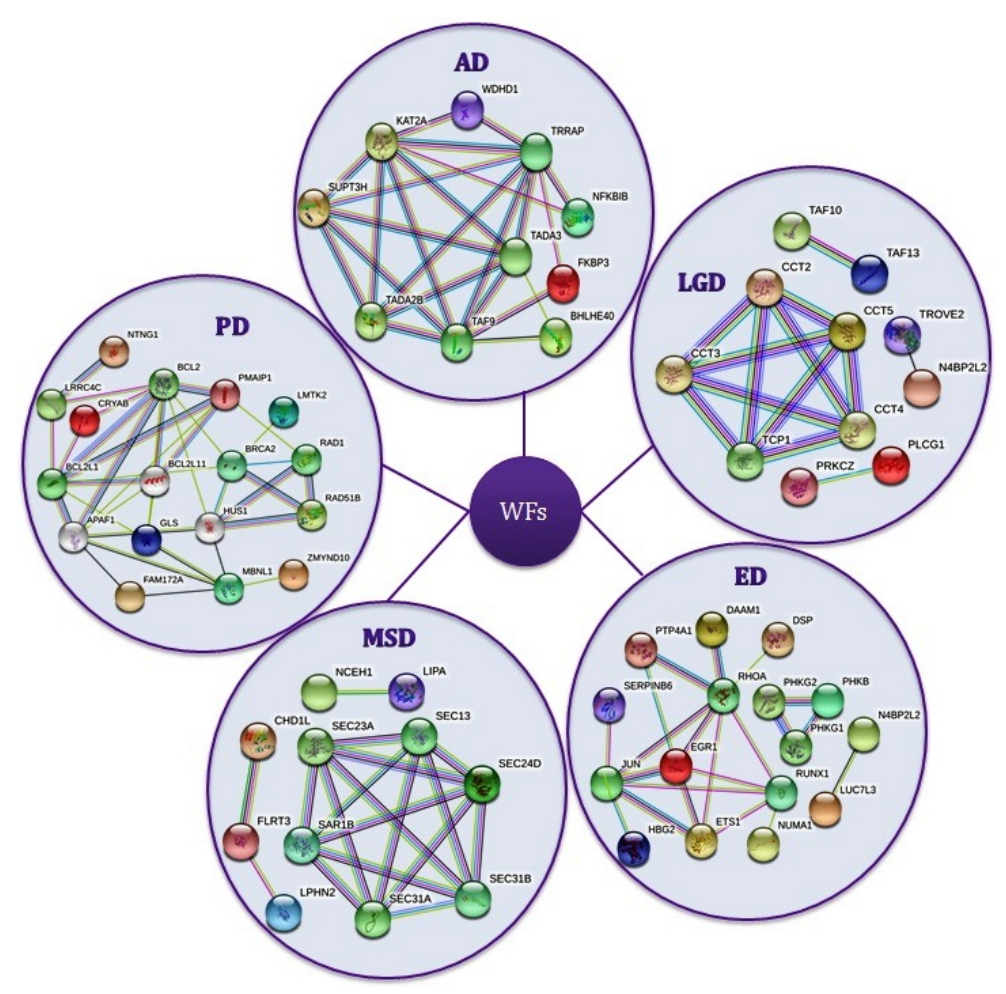

Figure 4: Protein-Protein Interaction Network of the Significantly Commonly Dysregulated Genes of the NDGDs with WFs.

other by underlying molecular or biological mechanisms. We identified pathways of the commonly differentially expressed genes (i.e. Dysregulated genes in between WFs and each NDGD) of each NDGD. These identified pathways agreed that WFs have a strong association with NDGDs. Similarly, gene expression ontologies and protein-protein interactions of common differentially expressed genes determine that WFs can carry several NDGDs on unsafe welder's health.

We have verified our result with the gold benchmark databases (dbGAP and OMIM) and found that there are some shared genes between the WFS and NDGDs as shown in figure 5. For cross checking the validity of our study, we collected genes and disease names from OMIM Disease, OMIM Expanded and dbGap databases using differentially expressed genes of WFs. We combined the diseases from three mentioned databases and selected only neurodegenerative diseases (NDGDs) after various steps of statistical analysis. Interestingly, we found our selected five NDGDs among the list of collected NDGDs from the mentioned databases as shown in figure 5. Therefore, it proved that WFs may have a strong association for the progression of PD, AD, LGD, ED and MSD neurodegenerative diseases $[7,13]$.

\section{Conclusions}

In this study, we have considered Gene Expression Omnibus (GEO) microarray data from welding fumes (WFs), Parkinson's disease (PD), Alzheimer's disease (AD), Lou Gehrig's dis- 


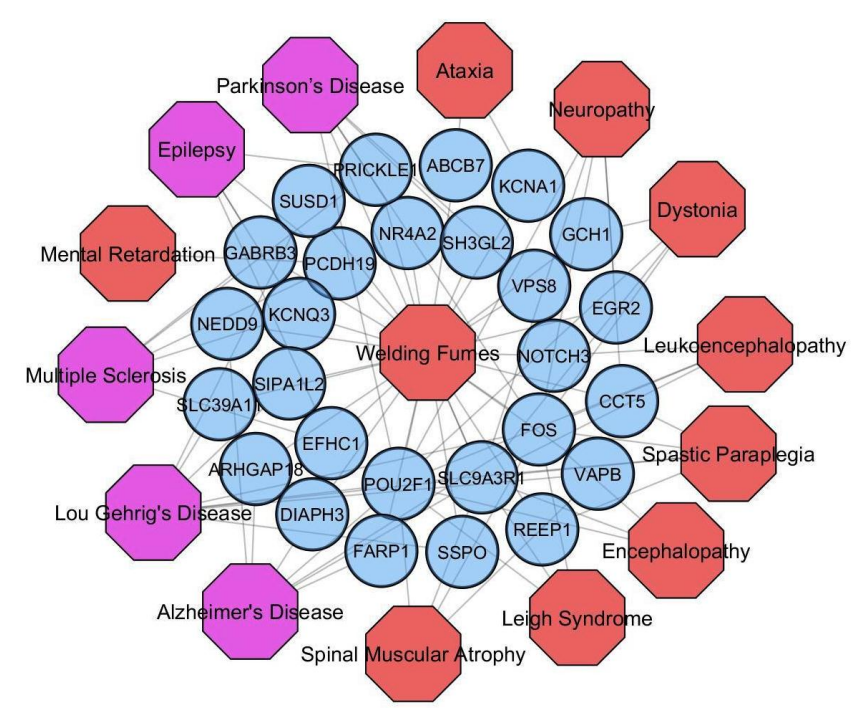

Figure 5: Disease network of Welding fumes (WFs) with several NDGDs. Red colored octagon-shaped nodes represent different categories of NDGDs, Violet colored octagon-shaped nodes represent our selected five NDGDs and round-shaped sky blue colored nodes represent differentially expressed genes for WFs. A link is placed between a disorder and a disease gene if mutations in that gene lead to the specific disorder.

ease (LGD), Epilepsy disease (ED), Multiple Sclerosis disease (MSD) and control datasets to analyze and investigate the genetic effects of WFs on neurodegenerative diseases (NDGDs). We analyzed dysregulated genes, disease relationship networks, dysregulated pathways, gene expression ontologies and protein-protein interactions of WFs and NDGDs. Our findings showed that WFs have a strong association with NDGDs. This kind of study will be useful for making genomic evidence based recommendations about the accurate disease prediction, identification and therapeutic treatments. This study also will be useful for making society aware of the dangerous effect of welding on the human body.

\section{References}

[1] J. M. Antonini, Health effects of welding, Critical reviews in toxicology 33 (1) (2003) 61-103.

[2] M. I. Khan, Welding science and technology, New Age International, 2007.

[3] K. America, Are there links between hazardous welding fumes and brain damage?, https:// kemperamerica.com/welding-fumes-brain-damage/, accessed: 2018-08-07 (2015).

[4] R. Sharma, S.-Y. Kim, A. Sharma, Z. Zhang, S. P. Kambhampati, S. Kannan, R. M. Kannan, Activated microglia targeting dendrimer-minocycline conjugate as therapeutics for neuroinflammation, Bioconjugate chemistry 28 (11) (2017) 2874-2886.

[5] M. Golpich, E. Amini, Z. Mohamed, R. Azman Ali, N. Mohamed Ibrahim, A. Ahmadiani, Mitochondrial dysfunction and biogenesis in neurodegenerative diseases: pathogenesis and treatment, CNS neuroscience \& therapeutics 23 (1) (2017) 5-22.

[6] W. Poewe, K. Seppi, C. M. Tanner, G. M. Halliday, P. Brundin, J. Volkmann, A.-E. Schrag, A. E. Lang, Parkinson disease, Nature reviews Disease primers 3 (2017) 17013.

[7] B. A. Racette, S. R. Criswell, J. I. Lundin, A. Hobson, N. Seixas, P. T. Kotzbauer, B. A. Evanoff, J. S. Perlmutter, J. Zhang, L. Sheppard, et al., Increased risk of parkinsonism associated with welding exposure, Neurotoxicology 33 (5) (2012) 1356-1361. 
[8] J. Wang, B. J. Gu, C. L. Masters, Y.-J. Wang, A systemic view of alzheimer diseaseinsights from amyloid- $\beta$ metabolism beyond the brain, Nature Reviews Neurology 13 (10) (2017) 612.

[9] E. Tokuda, Y. Furukawa, Abnormal protein oligomers for neurodegeneration, Oncotarget 8 (25) (2017) 39943.

[10] J. Sun, Commentary: target intestinal microbiota to alleviate disease progression in amyotrophic lateral sclerosis, Journal of neurology \& neuromedicine 2 (6) (2017) 13.

[11] M. Alves, E. Beamer, T. Engel, The metabotropic purinergic p2y receptor family as novel drug target in epilepsy, Frontiers in pharmacology 9 (2018) 193.

[12] K. Sapko, A. Szczepańska-Szerej, A. Jamroz-Wiśniewska, M. Kulczyński, M. Marciniec, K. Rejdak, Progressive forms of multiple sclerosis: disease-modifying therapy review, World Scientific News 105 (2018) 157-167.

[13] O. Proudfoot, et al., Manganese in manganism, parkinson's disease, huntington's disease, amyotrophic lateral sclerosis, and batten disease: A narrative review, Neurology India 65 (6) (2017) 1241.

[14] A. Stanam, Gene expression omnibus, https://www.ncbi.nlm.nih.gov/geo/query/acc.cgi?acc= GSE62384, accessed: 2018-08-08 (2018).

[15] N. M. Lewandowski, S. Ju, M. Verbitsky, B. Ross, M. L. Geddie, E. Rockenstein, A. Adame, A. Muhammad, J. P. Vonsattel, D. Ringe, et al., Polyamine pathway contributes to the pathogenesis of parkinson disease, Proceedings of the National Academy of Sciences 107 (39) (2010) 16970-16975.

[16] E. M. Blalock, H. M. Buechel, J. Popovic, J. W. Geddes, P. W. Landfield, Microarray analyses of laser-captured hippocampus reveal distinct gray and white matter signatures associated with incipient alzheimer's disease, Journal of chemical neuroanatomy 42 (2) (2011) 118-126.

[17] F. Dangond, D. Hwang, S. Camelo, P. Pasinelli, M. P. Frosch, G. Stephanopoulos, G. Stephanopoulos, R. H. Brown Jr, S. R. Gullans, Molecular signature of late-stage human als revealed by expression profiling of postmortem spinal cord gray matter, Physiological genomics 16 (2) (2004) 229-239.

[18] M. Carlet, K. Janjetovic, J. Rainer, S. Schmidt, R. Panzer-Grümayer, G. Mann, M. Prelog, B. Meister, C. Ploner, R. Kofler, Expression, regulation and function of phosphofructo-kinase/fructosebiphosphatases (pfkfbs) in glucocorticoid-induced apoptosis of acute lymphoblastic leukemia cells, BMC cancer 10 (1) (2010) 638.

[19] M. H. Han, D. H. Lundgren, S. Jaiswal, M. Chao, K. L. Graham, C. S. Garris, R. C. Axtell, P. P. Ho, C. B. Lock, J. I. Woodard, et al., Janus-like opposing roles of cd47 in autoimmune brain inflammation in humans and mice, Journal of Experimental Medicine (2012) jem-20101974.

[20] M. A. Moni, P. Lio, Genetic profiling and comorbidities of zika infection, The Journal of infectious diseases 216 (6) (2017) 703-712.

[21] M. A. Moni, P. Liò, comor: a software for disease comorbidity risk assessment, Journal of clinical bioinformatics 4 (1) (2014) 8.

[22] M. A. Moni, P. Liò, How to build personalized multi-omics comorbidity profiles, Frontiers in cell and developmental biology 3 (2015) 28.

[23] M. A. Moni, P. Liò, Network-based analysis of comorbidities risk during an infection: Sars and hiv case studies, BMC bioinformatics 15 (1) (2014) 333.

[24] L. Jin, X.-Y. Zuo, W.-Y. Su, X.-L. Zhao, M.-Q. Yuan, L.-Z. Han, X. Zhao, Y.-D. Chen, S.-Q. Rao, Pathway-based analysis tools for complex diseases: a review, Genomics, proteomics \& bioinformatics 12 (5) (2014) 210-220.

[25] M. V. Kuleshov, M. R. Jones, A. D. Rouillard, N. F. Fernandez, Q. Duan, Z. Wang, S. Koplev, S. L. Jenkins, K. M. Jagodnik, A. Lachmann, et al., Enrichr: a comprehensive gene set enrichment analysis web server 2016 update, Nucleic acids research 44 (W1) (2016) W90-W97.

[26] Reactome, Neuronal system (homo sapiens), https://reactome.org/content/detail/R-HSA210500, accessed: 2018-08-14 (2008).

[27] L. Sasset, Y. Zhang, T. M. Dunn, A. Di Lorenzo, Sphingolipid de novo biosynthesis: a rheostat of cardiovascular homeostasis, Trends in Endocrinology \& Metabolism 27 (11) (2016) 807-819.

[28] Wikipathways, Intrinsic pathway for apoptosis (homo sapiens), https://www.wikipathways.org/ index.php/Pathway:WP1841, accessed: 2018-08-14 (2018). 
[29] C. J. Lawrence, R. K. Dawe, K. R. Christie, D. W. Cleveland, S. C. Dawson, S. A. Endow, L. S. Goldstein, H. V. Goodson, N. Hirokawa, J. Howard, et al., A standardized kinesin nomenclature, The Journal of cell biology 167 (1) (2004) 19-22.

[30] Reactome, Circadian rhythm pathway, https://reactome.org/content/query?q=Circadian+ rhythm+pathway\&species=Homo+sapiens\&species=Entries+without+species\&cluster=true, accessed: 2018-08-14 (2008).

[31] A. J. Pratt, E. D. Getzoff, J. J. P. Perry, Amyotrophic lateral sclerosis: update and new developments, Degenerative neurological and neuromuscular disease 2012 (2) (2012) 1.

[32] Reactome, Signaling by hippo, https://reactome.org/content/detail/R-HSA-2028269, accessed: 2018-08-14 (2012).

[33] Wikipathways, p53 signaling pathway - homo sapiens (human), https://www.genome.jp/kegg-bin/ show_pathway?map=hsa04115\&show_description=show, accessed: 2018-08-15 (2018).

[34] S. Boros, R. J. Bindels, J. G. Hoenderop, Active ca2+ reabsorption in the connecting tubule, Pflügers Archiv-European Journal of Physiology 458 (1) (2009) 99-109.

[35] Bioportal, Gene regulation ontology, https://reactome.org/content/detail/R-HSA-2028269, accessed: 2018-08-15 (2016).

[36] Network analysis of protein interaction data: An introduction/ protein-protein interaction networks, https://www.ebi.ac.uk/training/onlkine/course/network-analysis-proteinprotein-interaction-data-introduction/, accessed: 2018-08-19 (2017).

[37] D. Szklarczyk, A. Franceschini, S. Wyder, K. Forslund, D. Heller, J. Huerta-Cepas, M. Simonovic, A. Roth, A. Santos, K. P. Tsafou, et al., String v10: protein-protein interaction networks, integrated over the tree of life, Nucleic acids research 43 (D1) (2014) D447-D452. 